\title{
Topology optimization in micromechanical resonator design
}

\author{
Wei He · David Bindel · Sanjay Govindjee
}

Received: 23 December 2009 / Accepted: 13 January 2011 / Published online: 8 February 2011

(C) The Author(s) 2011. This article is published with open access at Springerlink.com

\begin{abstract}
A topology optimization problem in micromechanical resonator design is addressed in this paper. The design goal is to control the first several eigenfrequencies of a micromechanical resonator using topology optimization. The design variable is the distribution of mass in a constrained domain which we model via (1) the Simple Isotropic Material with Penalization Model and (2) the Peak Function Model. The overall optimization problem is solved using the Method of Moving Asymptotes and a Genetic Algorithm combined with a local gradient method. A numerical example is presented to highlight the features of the methods in more detail. The advantages and disadvantages of each method are discussed.
\end{abstract}

Keywords Topology optimization · Micromechanical resonator · Eigen-frequency · Moving asymptotes $\cdot$ Genetic algorithm

\section{Introduction}

Micromechanical resonators are important elements in the design of on chip signal processing systems for many next generation communication and sensing devices; see, for example, Li et al. (2007). In the design of individual resonators, the control of eigen-frequencies and mode shapes of resonators plays a major role. Performance of the resonators, usually as a key part of a microelectromechanical system (MEMS), strongly relies on the accuracy of control of vibration modes. As pointed out in Bindel

\footnotetext{
W. He $(\bowtie) \cdot$ S. Govindjee

Department of Civil and Environmental Engineering, University of California, Berkeley, CA 94720, USA

e-mail: weihe@cal.berkeley.edu

D. Bindel

Department of Computer Science, Cornell University, Ithaca, NY 14853, USA
} 
and Govindjee (2005), it is desirable to control the distribution of eigen-frequencies in such systems (which are complex-valued), with better performances being obtained when the working frequencies (the real parts) are appropriately separated from each other. The control of this separation is essentially a topology optimization problem. So that we can focus on the main issue; we will confine our attention in this paper to non-damped systems-i.e., those with strictly real eigenvalues.

Topology optimization or generalized shape optimization of structures has been an active research area since at least the 1980s. One example that is frequently encountered in microelectromechanical system design is to find optimally compliant mechanisms (Saxena and Ananthasuresh 2000). The problem is usually to seek an optimal topology in a specified domain so that the structure can produce maximum displacements at some port. This is a typical optimization problem of specific entries of the global stiffness of a linearly elastic structure. A second important example occurs in car body design where optimal material layout is desired to reduce interior noise (Ma et al. 1993). This latter example is nearer to our area of interest as it involves the issue of dynamics.

The central task in topology optimization is to determine which geometric points in the design domain should be material points and which points should contain no material (i.e., are void). Based on a fine discretization by finite elements, one is faced with a large-scale " $0 / 1$ " type integer optimization problem. By employing a material interpolation function, one can transform the discrete problem into a continuous problem which is relatively easier to solve. First we will review three common material distribution models and later we will focus on the use of two of them within our problem context.

An important but somewhat cumbersome material distribution model is the homogenization method (Bendsoe 1989, 1995; Bendsoe and Kikuchi 1988). In this method, each material point is looked on as a composite material consisting of an infinite number of infinitely small holes and material points which are periodically distributed. The effective material elasticity tensor at a point is given by

$$
\boldsymbol{C}_{i j k l}(\boldsymbol{x})=\frac{1}{|Y|} \int_{Y}\left[\boldsymbol{C}_{i j k l}(\boldsymbol{x}, \boldsymbol{y})-\sum_{p q} \boldsymbol{C}_{i j p q}(\boldsymbol{x}, \boldsymbol{y}) \frac{\partial \chi_{p}^{k l}}{\partial y_{q}}\right] d \boldsymbol{y} .
$$

Here, $\chi_{p}^{k l}$ is the microscopic displacement field solution to the (six) variational cell equilibrium equations:

$$
\int_{Y} \sum_{p q i j} \boldsymbol{C}_{i j p q}(\boldsymbol{x}, \boldsymbol{y}) \frac{\partial \chi_{p}^{k l}}{\partial y_{q}} \frac{\partial v_{i}}{\partial y_{j}} d \boldsymbol{y}=\int_{Y} \sum_{i j} \boldsymbol{C}_{i j k l}(\boldsymbol{x}, \boldsymbol{y}) \frac{\partial v_{i}}{\partial y_{j}} d \boldsymbol{y} \quad \forall \boldsymbol{v},
$$

where $\boldsymbol{v}$ are $Y$-periodic displacement variations, $\boldsymbol{y}=\boldsymbol{x} / \epsilon$ represents fine scale positions and $\epsilon$ is a small parameter representing the ratio of fine scale to large scale feature sizes. This theory, via the distribution of holes in the composite, gives a functional relationship between the density of material in the composite and the effective material properties. With this at hand, an optimal distribution of mass in the composite material in the design domain can be computed and the result can be interpreted as an optimal topology by thresholding. 
Simple Isotropic Material with Penalization (SIMP) is a second common material interpolation model (Yin and Yang 2000). The elasticity tensor in this model is given by

$$
\boldsymbol{C}_{i j k l}(\boldsymbol{x})=[p(x)]^{\eta} \boldsymbol{C}_{i j k l}^{0}, \quad \eta>1,
$$

where $p(\boldsymbol{x})$, the design variable, is a density-like function and $\boldsymbol{C}_{i j k l}^{0}$ is the elasticity tensor of a given solid isotropic material. The parameter $\eta$ can be used to penalize intermediate densities. The advantage of the SIMP model is that we can avoid the microstructure analysis of the homogenization model and the simplicity of the model can facilitate design. It also has been pointed out that when $\eta$ is greater than or equal to three, the SIMP model obeys Hashin-Shtrikman bounds on the effective properties of composite materials (Yin and Yang 2000; Hashin and Shtrikman 1963).

The last model to be discussed here is the peak function model (Yin and Ananthasuresh 2001). In this model the elasticity interpolation is given as:

$$
\boldsymbol{C}_{i j k l}(\boldsymbol{x})=\sum_{m=1}^{n} \boldsymbol{C}_{i j k l}^{m} \exp \left[-\frac{\left[p(\boldsymbol{x})-\mu_{m}\right]^{2}}{2 \sigma_{m}^{2}}\right]+\boldsymbol{C}_{i j k l}^{v o i d},
$$

where $n$ is the number of material phases. With a small parameter $\sigma_{m}$, the exponential function in the expression is a continuous approximation to the $\delta$-function. The advantage of this model is that the design variable, $p(\boldsymbol{x})$, can take any value between $-\infty$ and $\infty$. Furthermore, this model can include multiple materials without increasing the number of design variables.

All these models have been successfully applied in the topology optimization of static problems, such as compliant mechanism design as mentioned above, tunnel support design, stiff structure design, etc. Further, the homogenization model has also been applied in the design of vibrating structures (Diaz and Kikuchi 1992; Kosaka and Swan 1999; Ma et al. 1995). The SIMP method can also be found to be used in eigenvalue optimization problems (Pedersen 2000; Tcherniak 2002). In this paper, the SIMP model and the peak function model will be explored for use in dynamic problems related to high frequency micromechanical resonator optimization.

Choosing appropriate optimization algorithms is another important issue in topology optimization. Due to a very large number of design variables, conventional mathematical programming methods may result in a very poor efficiency in topology optimization. As a result, a kind of Optimality Criteria (OC) updating algorithm is often used instead (Bendsoe and Kikuchi 1988; Suzuki and Kikuchi 1991). It solves the necessary conditions for an optimal point iteratively. Even though it converges well in static problems, it may not always work in dynamic problems. Thus some researchers have proposed a Modified Optimality Criteria (MOC) algorithm for frequency response optimization (Ma et al. 1993). This algorithm employs a shifted Lagrangian function to make a convex approximation and then solves the convex problem by using a dual method. In this paper, the Method of Moving Asymptotes (MMA) (Svanberg 1987) will be used as the main algorithm. MMA is based on a similar idea that deals with a non-convex problem by solving a sequence of convex approximations. It can be looked on as a further generalization of the widely used Convex Linearization method (CONLIN) (Fleury and Braibant 1986; 
Fleury 1989). In order to attempt to obtain a global optimum, Genetic Algorithms in conjunction with local refinement (Hybrid GA) will also be employed in this paper.

In summary, a simple but effective material distribution model and an efficient optimization algorithm along with a well-posed objective function form the key issues which must be addressed in topology optimization problems. In what follows we discuss our approach to each of these issues as they pertain to the central aspect of the problem of micromechanical resonator quality optimization-viz., the control of the real parts of the systems eigenvalues. As such, this paper focuses on the control of the first several eigen-frequencies of an undamped resonator.

\section{Material distribution models}

Two material interpolation models are used in this paper: one is the SIMP model and the other is the peak function model. As described in the previous section, the material interpolation function for the SIMP model is as follows:

$$
\boldsymbol{C}(\boldsymbol{x})=[p(\boldsymbol{x})]^{\eta_{1}} \boldsymbol{C}^{0},
$$

where $p$ is the design variable at point $\boldsymbol{x}$ and has a value between 0 and 1. To avoid singularities in finite element analysis, $p$ should have a lower bound which is slightly greater than 0 . The exponent $\eta_{1}$ is greater than 1 . For dynamics problems, a similar interpolation function is also needed for the mass density:

$$
\rho(\boldsymbol{x})=[p(\boldsymbol{x})]^{\eta_{2}} \rho^{0} .
$$

The only difference is that the exponent $\eta_{2}$ has a different value than $\eta_{1}$. The values of the two exponents need to be chosen so that the problem can be well approximated as the design variables approach their lower bound or upper bound.

For the peak function model, material interpolation functions for elastic moduli and mass density are defined as follows:

$$
\begin{aligned}
& \boldsymbol{C}(\boldsymbol{x})=\boldsymbol{C}^{0} \exp \left[-\frac{[p(\boldsymbol{x})]^{2}}{\sigma_{1}}\right]+\boldsymbol{C}^{\text {void }} \\
& \rho(\boldsymbol{x})=\rho^{0} \exp \left[-\frac{[p(\boldsymbol{x})]^{2}}{\sigma_{2}}\right]+\rho^{\text {void }} .
\end{aligned}
$$

Similarly, $\boldsymbol{C}^{\text {void }}$ and $\rho^{\text {void }}$ take on small positive values to avoid singularities. In this paper,

$$
\begin{aligned}
& \boldsymbol{C}^{\text {void }}=\boldsymbol{C}^{0} \times 10^{-15} \\
& \rho^{\text {void }}=\rho^{0} \times 10^{-16}
\end{aligned}
$$

and $\sigma_{1}$ and $\sigma_{2}$ are small positive parameters so that the functions act as $\delta$-functions. An advantage of the peak function model is that we do not need side constraints on the design variables. 


\section{Problem formulations}

The problem addressed here is to control the distribution of the (real) eigenfrequencies of a micromechanical resonator using topology optimization. In a finite element discretization, where the "density"-like design variables $p_{i}$ are element-wise constant and the subscript $i$ ranges from one to the number of elements in a specified design domain, nelt, the eigen-frequencies of the structure are functions of $p_{i}$. In order to unify the two material distribution models, let us define another element-wise constant quantity:

$$
\theta_{i}=p_{i}^{\eta_{1}}, \quad i=1, \text { nelt }
$$

for the SIMP model and

$$
\theta_{i}=\exp \left[-\frac{p_{i}^{2}}{\sigma_{1}}\right], \quad i=1, \text { nelt }
$$

for the peak function model. Then the general problem can be formulated as:

$$
\min _{\substack{p_{i} \\ i=1, \text { nelt }}} \sum_{k=1}^{n}\left|\frac{\omega_{k}\left(p_{i}\right)-\bar{\omega}_{k}}{\bar{\omega}_{k}}\right|
$$

subject to

$$
g=\sum_{i=1}^{\text {nelt }} \theta_{i}\left(1-\theta_{i}\right)=0 .
$$

The constraint implies that in each element the quantity $\theta_{i}$ must be either 0 or 1 .

For the SIMP model, we further need to add bounds on the design variables:

$$
0.05 \leq p_{i} \leq 1, \quad i=1, \text { nelt }
$$

where 0.05 is used instead of 0 for lower bound to avoid singularities. It should be noted that due to the lower bound on the design variables in the SIMP model or the definition of $\theta_{i}$ in the peak function model, the constraint equation (14) can only be approximately satisfied as $\theta_{i}$ can never be exactly equal to 0 . In the objective function, the $\bar{\omega}_{k}$ are pre-specified target eigen-frequencies. By adjusting $\bar{\omega}_{k}$, we can cluster certain eigen-frequencies or make them well separated.

With this type of objective function, it is possible to obtain non-physically realizable checkerboard topologies. In order to avoid checkerboard patterns or one-node connected hinges in structures if four-node square finite elements are used, we need to add another constraint, as done in Poulsen (2002):

$$
H(\boldsymbol{p})=0,
$$

where

$$
H(\boldsymbol{p})=\sum_{i, j} h\left(\theta_{i, j}, \theta_{i+1, j}, \theta_{i, j+1}, \theta_{i+1, j+1}\right)
$$


is a descriptor function for one-node connected hinges. Here the subscripts of $\theta$ imply the four elements topologically connected to the node examined and the sum runs over all nodes. The local function

$$
h(a, b, c, d)=m(a, b, d) \times m(a, c, d) \times m(b, a, c) \times m(b, d, c)
$$

is defined at each node and

$$
m(a, b, c)=|b-a|+|c-b|-|c-a| .
$$

If we assume that $\theta_{i}$ has reached either 0 or 1 in each element, then each one-node connected hinge will make the local function $h=16$. Note that local functions are non-negative and they can never be canceled out from node to node.

In summary, the problem formulation consists of (13), (14), (16). If the SIMP model is used, the constraint shown in (15) should be included as well.

\section{Optimization algorithms}

Two types of algorithms will be used to solve the optimization problems posed above. One is a Genetic Algorithm and the other is the Method of Moving Asymptotes (MMA) (Svanberg 1987, 1995; Bruyneel et al. 2002). Due to the probable large size of the topology optimization problem, the conventional Genetic Algorithm must be combined with local refinement by gradient methods (Hybrid GA) to improve efficiency. In this paper, Hybrid GA is applied in conjunction with the peak function model. The constraint condition is satisfied using a penalty method. The problem formulation is then slightly modified as:

$$
\min _{\substack{p_{i} \\ i=1, \text { nelt }}} \sum_{k=1}^{n}\left|\frac{\omega_{k}\left(p_{i}\right)-\bar{\omega}_{k}}{\bar{\omega}_{k}}\right|+w_{1} \times g+w_{2} \times H .
$$

The performance of the penalty method depends strongly on the choice of penalty factor and this actually constrains the wide application of the method, especially when it is hard to predict the ultimate optimal value for each term. However, in our case it is not a problem because we clearly know how large each term on the right hand side of (20) should be in a useful design. The acceptable value of the summation term is determined based on the accepted tolerance in the problem. In other words, it depends on how closely the target eigen-frequencies are going to be approached. The second term $g$ should be almost zero and the third term $H$ must be zero; i.e., the two constraints should be strictly met. The penalty factors in this paper were chosen as:

$$
\begin{aligned}
& w_{1}=10^{5} \\
& w_{2}=1 .
\end{aligned}
$$

In general, the factors should be chosen such that the values of $w_{1} \times g$ and $w_{2} \times H$ are comparable to ensure that violation of either constraint would lead to substantial change in the objective function value. 
GA here is combined with the standard gradient optimization method BFGS for local refinement. One or two iterations of BFGS were applied to each sampling point of GA first and then GA evolutions start from the local optimums obtained by BFGS earlier. This can greatly improve the performance of GA and save on overall compute time. In this paper, the BFGS algorithm was implemented using MATLAB's built-in function "fminunc" and GA was implemented using MATLAB code from Houck et al. (1995).

The second algorithm used here is MMA, which was particularly developed for structural optimization. It solves a sequence of convex approximating subproblems. Due to their convexity and separability, the subproblems can be efficiently solved by a dual method. This makes MMA a good choice for large scale problems in topology optimization. Furthermore, unlike the penalty method above, the dual method does not need user to define penalty factors to take care of constraint conditions. In our case, MMA is applied along with the SIMP model. The formulation was slightly modified as:

$$
\min _{\substack{p_{i} \\ i=1, \text { nelt }}} \sum_{k=1}^{n}\left|\frac{\omega_{k}\left(p_{i}\right)-\bar{\omega}_{k}}{\bar{\omega}_{k}}\right|
$$

subject to

$$
\begin{aligned}
& g+H=0 \\
& 0.05 \leq p_{i} \leq 1, \quad i=1, \text { nelt } .
\end{aligned}
$$

Again, the constraint equation (24) can only be approximately satisfied as previously explained. One difficulty in topology optimization is that the nearest local minima have to be avoided (Sigmund and Petersson 1998; Sigmund 1997). Local minima may make the MMA iteration stop very quickly and return an unacceptable result. Some techniques have been developed to deal with the problem (Sigmund and Petersson 1998; Sigmund 1997; Hilding 2000). The technique used here is a heuristic homotopy one. The constraint was relaxed first and then gradually made strict. As a result, many (non-feasible) intermediate "densities" would appear in the beginning of the optimization process, but this allowed the algorithm to avoid local minima and achieve better results to some extent. As the feasible domain becomes exact, all the intermediate "densities" go to their limiting values, either 0 or 1.

\section{Sensitivity analysis}

Both Hybrid GA and MMA require efficient gradient evaluations. Fortunately, for the problem which we address the gradient can be computed analytically. For the derivative of eigen-frequencies, consider the following standard eigen problem:

$$
\left(\boldsymbol{K}-\lambda_{n} \boldsymbol{M}\right) \phi_{n}=0
$$

where $\lambda_{n}=\omega_{n}^{2}$ and $\boldsymbol{K}$ and $\boldsymbol{M}$ are global stiffness and mass matrices from a finite element discretization. The eigen-vectors will be assumed to be mass orthonormal. 
The sensitivity of $\lambda_{n}$ to design variable $p_{i}$ can be written as (Ma et al. 1995; Ma and Hagiwara 1991):

$$
\frac{\partial \lambda_{n}}{\partial p_{i}}=\boldsymbol{\phi}_{n}^{T}\left(\frac{\partial \boldsymbol{K}}{\partial p_{i}}-\lambda_{n} \frac{\partial \boldsymbol{M}}{\partial p_{i}}\right) \boldsymbol{\phi}_{n}=\boldsymbol{\phi}_{n, i}^{T}\left(\frac{\partial \boldsymbol{k}_{i}}{\partial p_{i}}-\lambda_{n} \frac{\partial \boldsymbol{m}_{i}}{\partial p_{i}}\right) \boldsymbol{\phi}_{n, i},
$$

where $\phi_{n, i}$ stands for the component of the $n$th eigen-vector pertaining to the $i$ th finite element and $\boldsymbol{k}_{i}$ and $\boldsymbol{m}_{i}$ denote the element stiffness and mass matrices, respectively. The sensitivity of the element stiffness and mass matrices can be calculated in a straight-forward manner:

$$
\begin{aligned}
\frac{\partial \boldsymbol{k}_{i}}{\partial p_{i}} & =\int_{\Omega_{i}} \boldsymbol{B}_{i}^{T} \frac{\partial \boldsymbol{D}_{i}}{\partial p_{i}} \boldsymbol{B}_{i} d \Omega \\
\frac{\partial \boldsymbol{m}_{i}}{\partial p_{i}} & =\int_{\Omega_{i}} \frac{\partial \rho_{i}}{\partial p_{i}} \boldsymbol{N}_{i}^{T} \boldsymbol{N}_{i} d \Omega .
\end{aligned}
$$

For plane stress problems, the material matrix in the $i$ th element is given as:

$$
\boldsymbol{D}_{i}=\left[\begin{array}{ccc}
\frac{2 \lambda^{i} \mu^{i}}{\lambda^{i}+2 \mu^{i}}+2 \mu^{i} & \frac{2 \lambda^{i} \mu^{i}}{\lambda^{i}+2 \mu^{i}} & 0 \\
\frac{2 \lambda^{i} \mu^{i}}{\lambda^{i}+2 \mu^{i}} & \frac{2 \lambda^{i} \mu^{i}}{\lambda^{i}+2 \mu^{i}}+2 \mu^{i} & 0 \\
0 & 0 & \mu^{i}
\end{array}\right],
$$

where $\lambda^{i}$ and $\mu^{i}$ are the Lame modulus and the shear modulus of material in the $i$ th element, respectively.

For the SIMP model

$$
\begin{aligned}
& \lambda^{i}=\lambda^{0} p_{i}^{\eta_{1}} \\
& \mu^{i}=\mu^{0} p_{i}^{\eta_{1}} \\
& \rho^{i}=\rho^{0} p_{i}^{\eta_{2}}
\end{aligned}
$$

and

$$
\begin{aligned}
\frac{\partial \boldsymbol{D}_{i}}{\partial p_{i}} & =\frac{\eta_{1}}{p_{i}} \boldsymbol{D}_{i} \\
\frac{\partial \rho_{i}}{\partial p_{i}} & =\frac{\eta_{2}}{p_{i}} \rho_{i} .
\end{aligned}
$$

For the peak function model

$$
\begin{aligned}
& \lambda^{i}=\lambda^{0} \exp \left[-\frac{p_{i}^{2}}{\sigma_{1}}\right]+\lambda^{\text {void }} \\
& \mu^{i}=\mu^{0} \exp \left[-\frac{p_{i}^{2}}{\sigma_{1}}\right]+\mu^{\text {void }} \\
& \rho^{i}=\rho^{0} \exp \left[-\frac{p_{i}^{2}}{\sigma_{2}}\right]+\rho^{\text {void }}
\end{aligned}
$$


and

$$
\begin{aligned}
\frac{\partial \boldsymbol{D}_{i}}{\partial p_{i}} & =-\frac{2 p_{i}}{\sigma_{1}} \boldsymbol{D}_{i} \\
\frac{\partial \rho_{i}}{\partial p_{i}} & =-\frac{2 p_{i}}{\sigma_{2}} \rho_{i},
\end{aligned}
$$

where the constant terms with superscript "void" do not affect the sensitivities, and again the terms with superscript " 0 " are elasticity moduli and mass density of the given solid isotropic material. It can be seen that the gradients of the stiffness matrix and the mass matrix can be simply expressed as linear function of themselves.

It should be noted that the sensitivity calculation is different for repeated eigenfrequencies. In fact, the sensitivity analysis for repeated eigenvalues and the associated eigenmodes has been discussed frequently (Chen and Pan 1986; Dailey 1989; Juang et al. 1989; Mills-Curran 1988; Ma and Hagiwara 1994; Kenny and Hou 1994). Assume that $\phi_{1}$ and $\phi_{2}$ are a pair of eigenvectors associated with the (doubly) repeated eigenvalue $\lambda$. Then any linear combination of $\boldsymbol{\phi}_{1}$ and $\boldsymbol{\phi}_{2}, y_{1} \boldsymbol{\phi}_{1}+y_{2} \boldsymbol{\phi}_{2}$, is also an eigenvector associated with $\lambda$. The derivatives of a repeated eigenvalue $\lambda$ can be obtained as the solution to the new $(2 \times 2)$ eigenvalue problem:

$$
\left(\overline{\boldsymbol{K}}-\frac{\partial \lambda_{j}}{\partial p_{i}} \overline{\boldsymbol{M}}\right) \boldsymbol{y}_{j}=0
$$

where

$$
\begin{aligned}
\overline{\boldsymbol{K}} & =\left[\boldsymbol{\phi}_{1}, \boldsymbol{\phi}_{2}\right]^{T}\left(\frac{\partial \boldsymbol{K}}{\partial p_{i}}-\lambda \frac{\partial \boldsymbol{M}}{\partial p_{i}}\right)\left[\boldsymbol{\phi}_{1}, \boldsymbol{\phi}_{2}\right] \\
\overline{\boldsymbol{M}} & =\left[\boldsymbol{\phi}_{1}, \boldsymbol{\phi}_{2}\right]^{T} \boldsymbol{M}\left[\boldsymbol{\phi}_{1}, \boldsymbol{\phi}_{2}\right]
\end{aligned}
$$

and $\boldsymbol{y}_{j}=\left[y_{1}, y_{2}\right]^{T}$ is a $2 \times 1$ vector.

We further need to calculate the sensitivities of the descriptor function which has been addressed in Poulsen (2002). Consider the local function $h$ defined in (18), its derivative with respect to the 1 st entry can be written as:

$$
\begin{aligned}
\frac{\partial h}{\partial a}= & \frac{\partial m(a, b, d)}{\partial a} \times m(a, c, d) \times m(b, a, c) \times m(b, d, c) \\
& +\frac{\partial m(a, c, d)}{\partial a} \times m(a, b, d) \times m(b, a, c) \times m(b, d, c) \\
& +\frac{\partial m(b, a, c)}{\partial a} \times m(a, b, d) \times m(a, c, d) \times m(b, d, c) .
\end{aligned}
$$

Then the sensitivity of $H$ to $\theta_{i, j}$ is:

$$
\frac{\partial H}{\partial \theta_{i, j}}=\sum_{i=\max (i-1,1)}^{\min \left(i, n_{1}-1\right)} \sum_{j j=\max (j-1,1)}^{\min \left(j, n_{2}-1\right)} h_{, k}\left(\theta_{i i, j j}, \theta_{i i+1, j j}, \theta_{i i, j j+1}, \theta_{i i+1, j j+1}\right),
$$

where

$$
k=i-i i+2(j-j j)+1
$$


and $h_{, k}$ stands for the derivative with respect to the $k$ th argument. Here it is assumed that the design domain is discretized into $n_{1} \times n_{2}$ square four-node finite elements. The sensitivity of $H$ to design variable $p_{i}$ can then be calculated by using the chain rule.

The sensitivity analysis of constraint (14) is trivial and omitted here.

\section{Numerical examples}

\subsection{Description of problem}

The example problem considered in this paper is the design of a square bounded micromechanical resonator. This example is a model for a very general type of design problem in which a resonator with a set of desired frequencies must fit in a limited area of chip space. The topology of the resonator has to be determined to meet those design requirements. The main goal in this example is to adjust the first several eigenfrequencies using topology optimization. The white part in the center of Fig. 1 is the design domain. The blue part represents existing material and is fixed at its periphery. The material of the resonator is poly-silicon, with the following properties:

$$
\begin{aligned}
E & =150 \mathrm{GPa} \\
v & =0.226 \\
\rho & =2330 \mathrm{~kg} / \mathrm{m}^{3} .
\end{aligned}
$$

The structure has an in-plane vibration mode and a plane stress solid model is assumed in the whole structure. Four-node bi-linear elements were used to discretize the domain. The finite element analysis was performed using HiQLab (Bindel 2005). After discretizing the design domain into finite elements, one needs to determine the material density in each element. In other words, one needs to determine which elements are void and which are filled.

\subsection{Validity of material distribution models}

The validity of the material distribution models was examined first. For the models to be physically useful, the parameters $\eta_{1}, \eta_{2}$ in the SIMP model or $\sigma_{1}, \sigma_{2}$ in the peak function model must be chosen so that as $\theta$ goes to the limiting values 0 or 1 , the resulting eigen-frequencies approach those from a pure discrete problem. Though this is guaranteed by the distribution models when $\theta$ goes to 1 , it is not necessarily the case when $\theta$ goes to 0 because both stiffness and mass matrices approach 0 simultaneously and the resulting eigen-frequencies could be very different from the corresponding discrete problem. To avoid that, the parameters should be set such that the ratio between $\eta_{1}$ and $\eta_{2}$ in the SIMP model and between $\sigma_{1}$ and $\sigma_{2}$ in the peak function model are in proper ranges. The working values usually can be obtained after several trials. In addition, due to the lower bound on design variables in the SIMP model, $\eta_{1}$ and $\eta_{2}$ must be large enough to ensure that material density can approach 0 . 


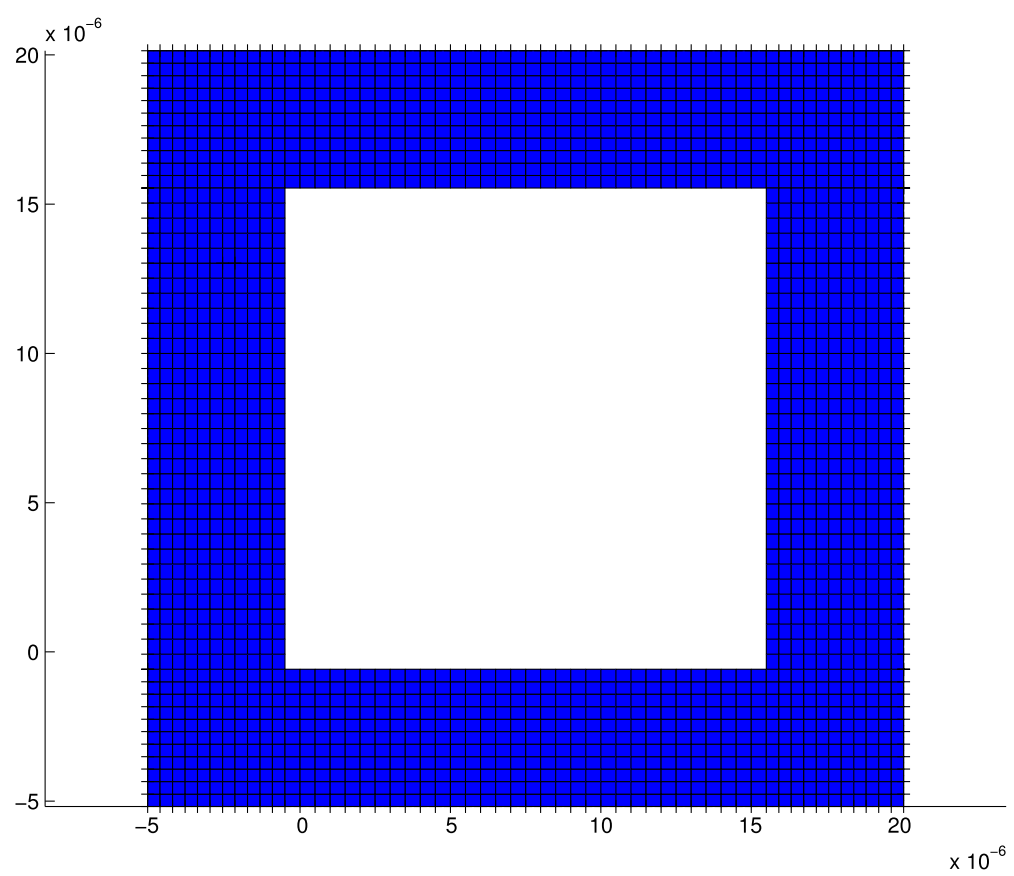

Fig. 1 Design domain of a micromechanical resonator (interior white part). Blue part represents existing material. Dimensions are in meters

Fig. 2 Convergence of SIMP model (solid lines), with pure discrete model used when $\theta=0$ and $\theta=1$ (markers). From $\theta=0$ to $\theta=1$, the first 5 eigen-frequencies change from (2.04 GHz, $2.07 \mathrm{GHz}$, $2.07 \mathrm{GHz}, 2.11 \mathrm{GHz}, 2.63 \mathrm{GHz})$ to $(1.18 \mathrm{GHz}, 1.18 \mathrm{GHz}$, $1.46 \mathrm{GHz}, 1.74 \mathrm{GHz}, 1.98 \mathrm{GHz}$ )

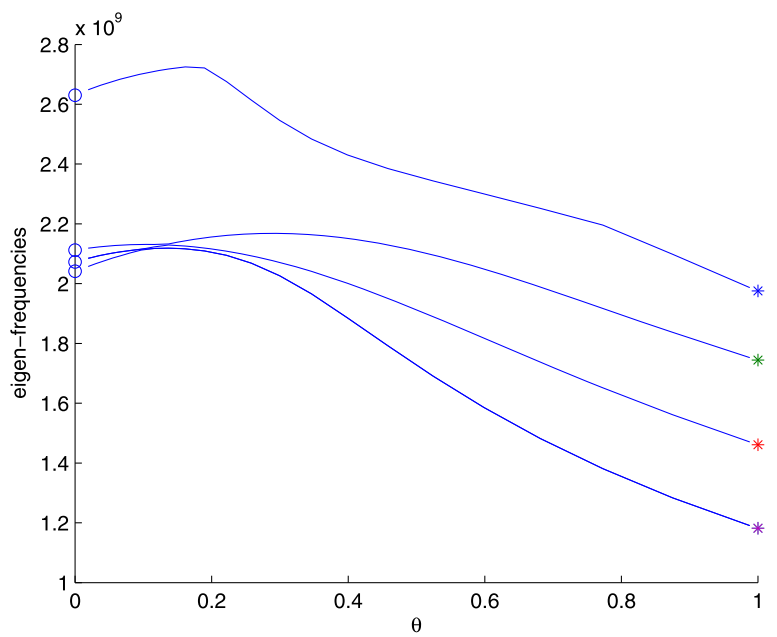

In this paper these parameters were set as:

$$
\begin{aligned}
& \eta_{1}=6, \quad \eta_{2}=11 \\
& \sigma_{1}=0.01, \quad \sigma_{2}=0.005 .
\end{aligned}
$$


Fig. 3 Convergence of peak function model (solid lines), with pure discrete model used when $\theta=0$ and $\theta=1$ (markers). From $\theta=0$ to $\theta=1$, the first 5 eigen-frequencies change from $(2.04 \mathrm{GHz}$, $2.07 \mathrm{GHz}, 2.07 \mathrm{GHz}, 2.11 \mathrm{GHz}$, $2.63 \mathrm{GHz})$ to $(1.18 \mathrm{GHz}$,

$1.18 \mathrm{GHz}, 1.46 \mathrm{GHz}, 1.74 \mathrm{GHz}$, $1.98 \mathrm{GHz})$

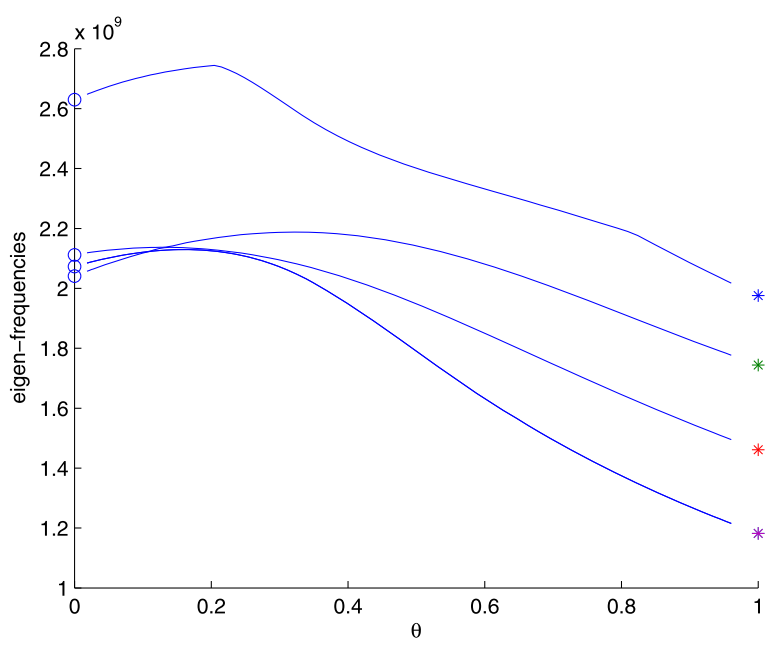

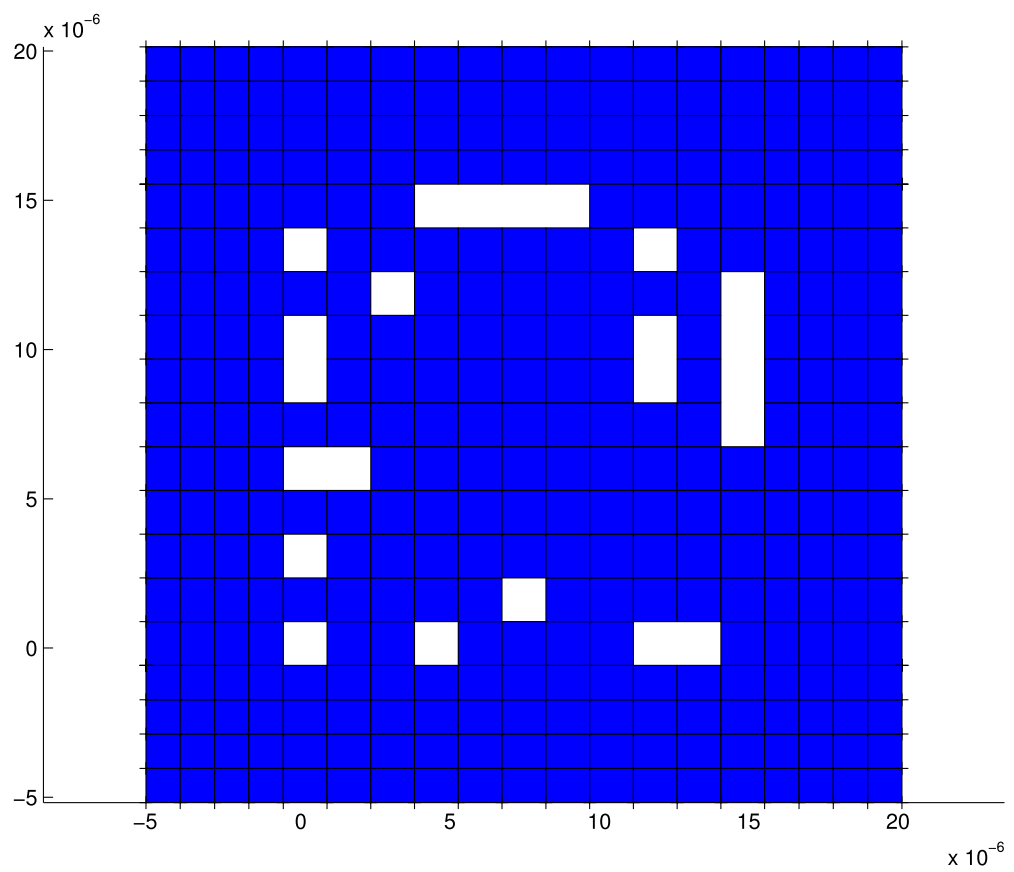

Fig. 4 Optimal topology for clustering the first 2 eigen-frequencies around $1.04 \mathrm{GHz}$ by Hybrid GA (irregular but accurate in terms of objective function value). Dimensions are in meters

In Figs. 2 and 3, the first 5 eigen-frequencies of the domain shown in Fig. 1 were calculated from a pure discrete model for the cases of $\theta=0$ (i.e., the interior part is void, with the resulting first 5 eigen-frequencies: $2.04 \mathrm{GHz}, 2.07 \mathrm{GHz}, 2.07 \mathrm{GHz}$, 

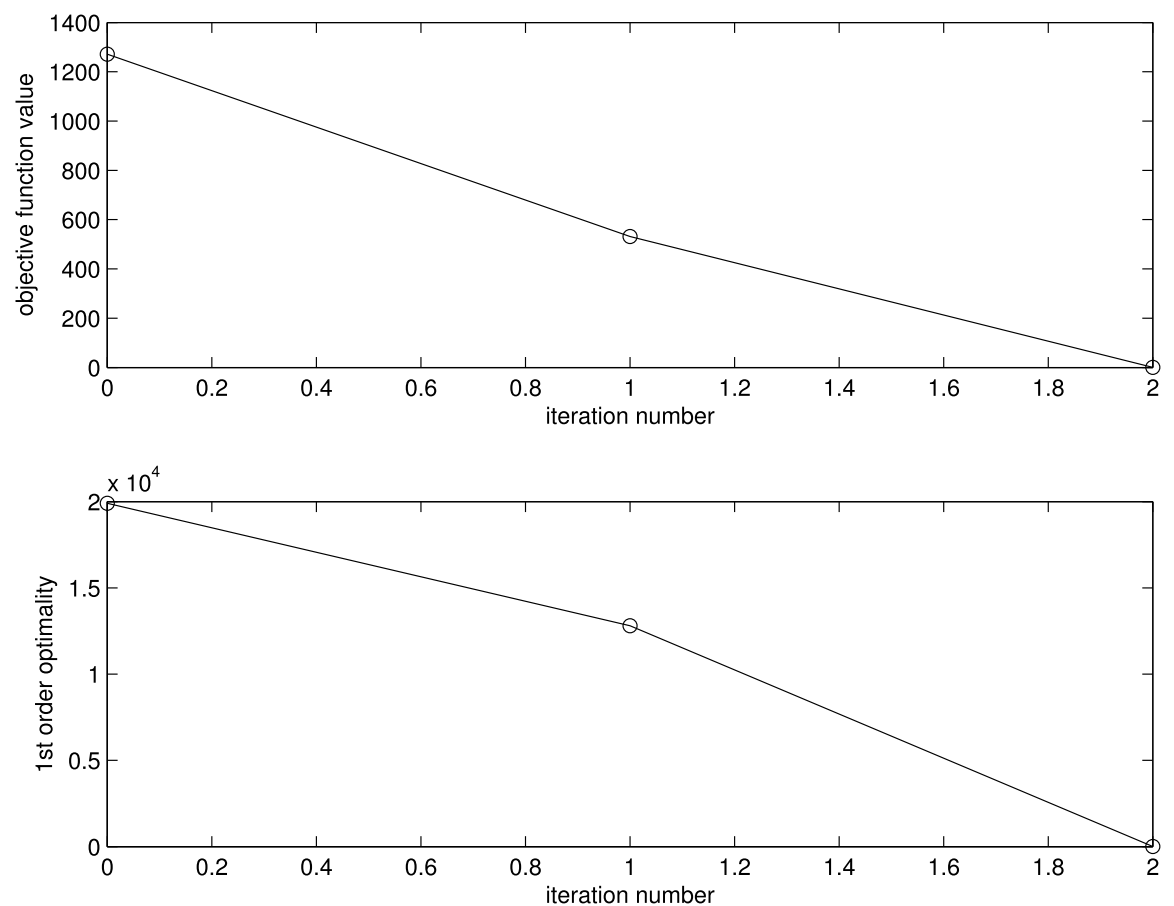

Fig. 5 Efficiency of local refinement in Hybrid GA, with both objective function value (upper graph) and first order optimality (lower graph) going down significantly after two iterations of BFGS

Table 1 Comparison between GA and Hybrid-GA

\begin{tabular}{lll}
\hline & Conventional GA & Hybrid-GA \\
\hline Function evaluations & 11817 & 1060 \\
Generations & 500 & 10 \\
Compute time & $350 \mathrm{~min}$ & $115 \mathrm{~min}$ \\
Objective function value attained & 0.072 & 0.010 \\
\hline
\end{tabular}

$2.11 \mathrm{GHz}, 2.63 \mathrm{GHz}$ ) and $\theta=1$ (i.e., the interior part is completely filled, with the resulting first 5 eigen-frequencies: $1.18 \mathrm{GHz}, 1.18 \mathrm{GHz}, 1.46 \mathrm{GHz}, 1.74 \mathrm{GHz}$, $1.98 \mathrm{GHz}$ ), otherwise they were calculated from the continuous mass distribution models. Note that the case of $\theta=0$ produces the eigenvalues solely associated with the existing boundary material. We can see that the continuous mass distribution models converge well to the two limiting cases. This implies that each element can be simply interpreted as "on" or "off" as long as material density in it is close to 1 or 0.

\subsection{Results by Hybrid GA}

As the main advantage, Hybrid GA can find global optima, but it is still expensive as compared to MMA due to a very large number of objective function evaluations. As mentioned before, in this paper Hybrid GA is applied in conjunction with the peak 

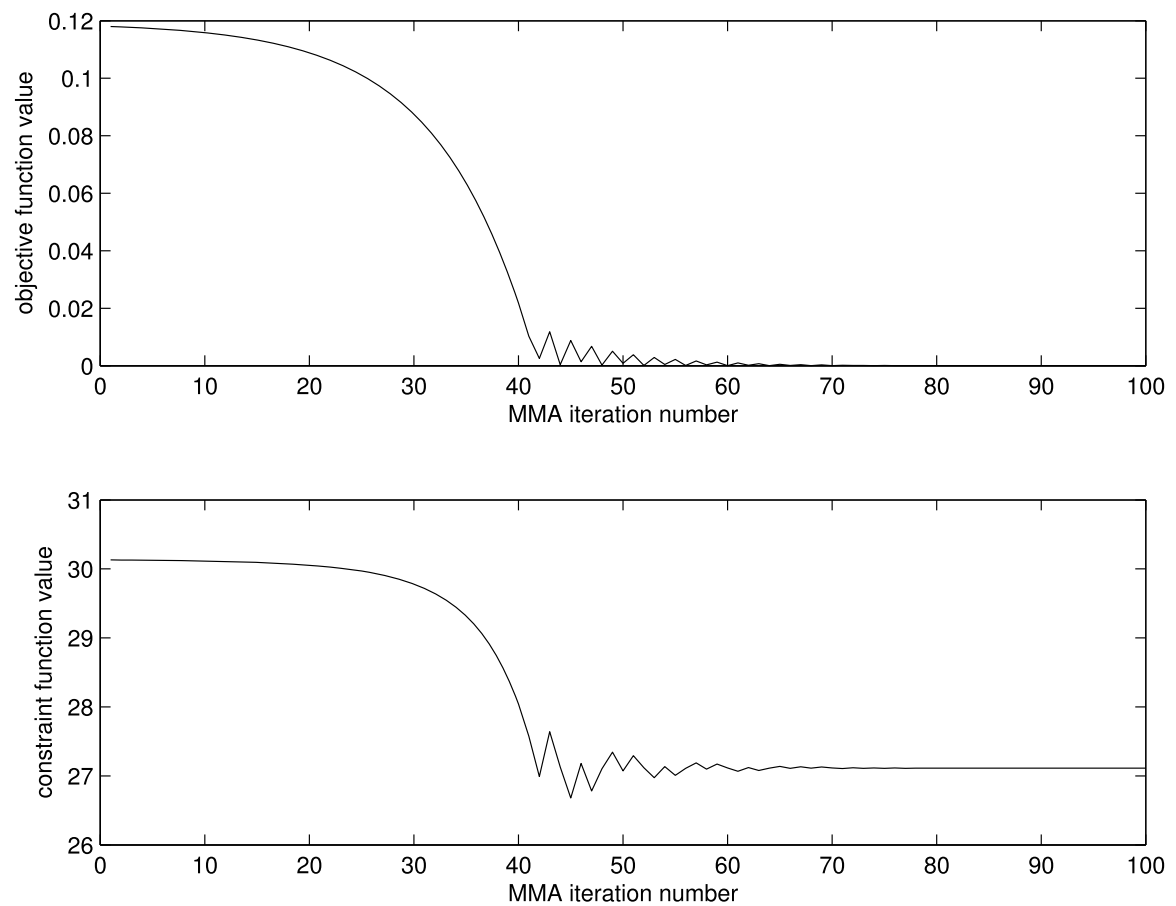

Fig. 6 First constrained problem solved by MMA, with small objective function value attained (upper graph) but large constraint function value (lower graph)

function material model. It can be applied on a coarse mesh resolution to get a preliminary result. Figure 4 shows an optimal topology obtained by Hybrid GA, where the goal was to cluster the first two eigen-frequencies around $1.04 \mathrm{GHz}$ and the design domain was discretized into 121 finite elements. We obtained $\omega_{1}=1.0400 \mathrm{GHz}$ and $\omega_{2}=1.0434 \mathrm{GHz}$. The result is very good in terms of frequencies, but it demonstrates an irregular topology and is certainly not good from the point of view of manufacturing. Compared to a numerical example shown later, it will be seen that the hybrid GA can generate better objective function values.

\subsection{Utility of local refinement in Hybrid GA}

The efficiency of local refinement in Hybrid GA was examined as well. Starting from each sampling point in the previous example, one or two BFGS iterations were conducted. Figure 5 shows that local optimization strongly improves the efficiency of GA. Both the objective function value and 1st order optimality condition decrease significantly in only five or six function evaluations, thus greatly saving overall compute time. Table 1 shows a comparison of the overall performance between conventional GA and Hybrid GA when the design domain is discretized into 676 elements. 

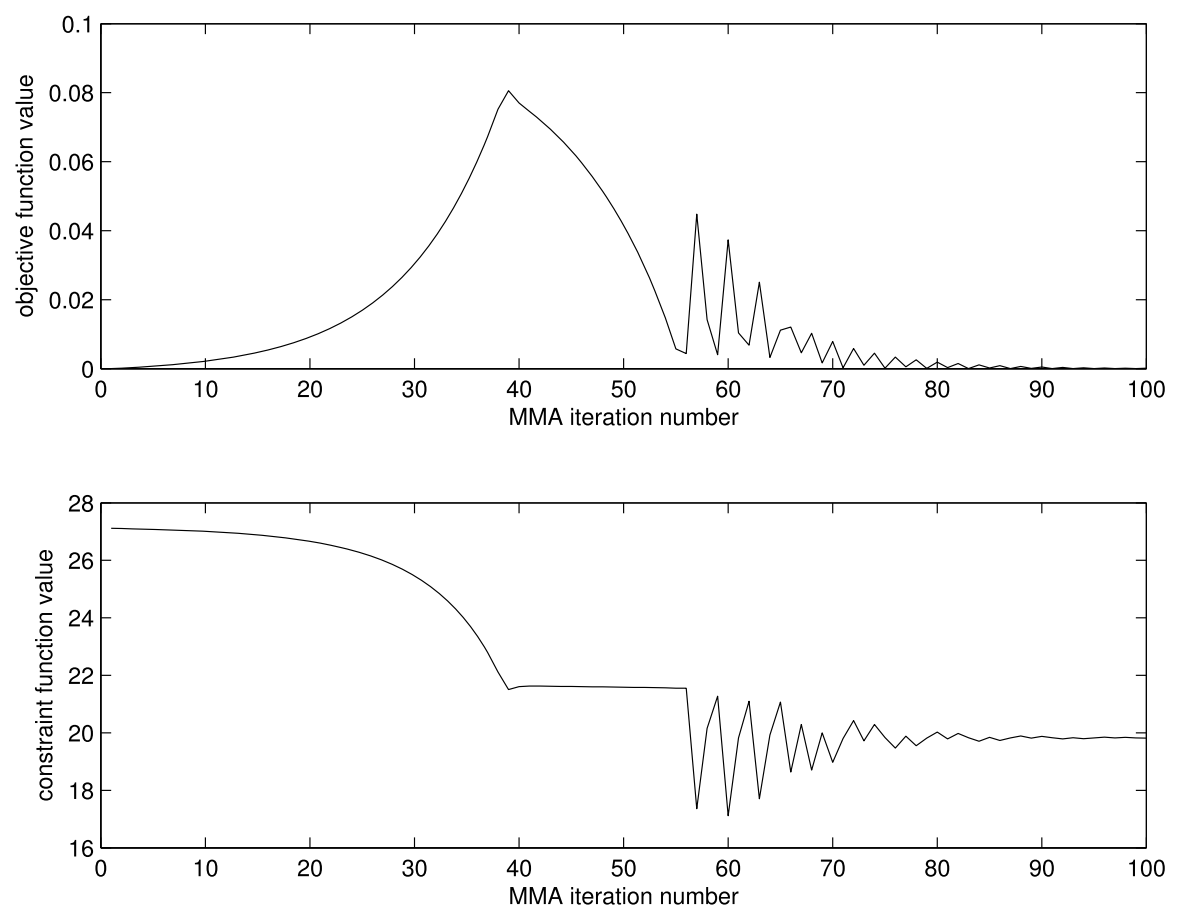

Fig. 7 Second constrained problem solved by MMA, with constraint function value going down (lower graph) but objective function value going up a little (upper graph)

\subsection{Results by MMA}

As mentioned before, in this paper MMA is applied together with the SIMP material model. MMA is a local optimization algorithm, so it is prone to getting stuck in local minima. To avoid this, we have used a kind of heuristic homotopy method mentioned above. By firstly applying a relaxed constraint and then making it stricter and stricter, instead of directly enforcing the exact constraint condition, one can achieve a satisfactory optimal design. In this case, the constraint function in (24) is rewritten as

$$
g+H \leq \epsilon .
$$

In the very beginning of the optimization process, $\epsilon$ is given a large positive value so that the constraint is essentially redundant. In the next step, the value of $\epsilon$ is lowered and a new sequence of MMA iterations is performed again to solve the new and more strictly constrained problem. We repeat this procedure until the original constraint is satisfied, which means that all the intermediate variables get very close to their limiting values 0 or 1 . Figure 6 shows how MMA works in the first step when the constraint condition is relatively weak; the upper curve plots the objective function values versus iteration number while the lower curve plots the constraint function values during the iterations. Figure 7 shows the same information in the following step, where a smaller value of $\epsilon$ makes the optimal point in the first step infeasible. 

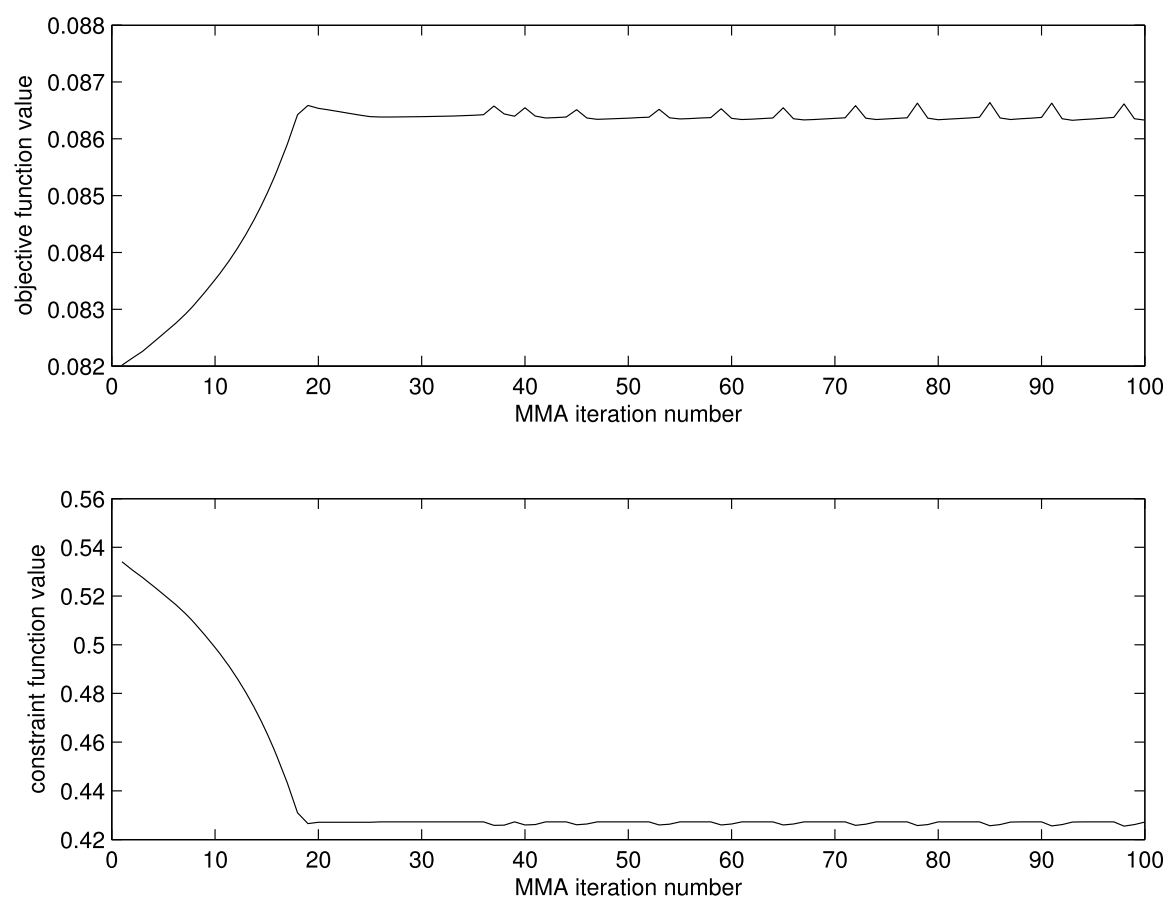

Fig. 8 Last constrained problem solved by MMA, with both objective function value (upper graph) and constraint function value being small (lower graph)

It will then converge to a new optimal point from outside the feasible space defined by the current $\epsilon$. During this period, the objective function value has to go up but the constraint function value goes down. In Fig. 7 it eventually stops at an interior point near the boundary. After solving a sequence of constrained optimization problems we can obtain a good optimal design. Figure 8 shows the terminal step where the objective function value can not go down inside the feasible domain, which implies that it converges to an optimal point that lies on the boundary. Both objective function value and constraint function value are reasonably small at the end of the process.

Some optimal topologies obtained using MMA are shown below. In these numerical examples there are 1024 elements in the design domain. The related parameters used in our MMA had the following value for each variable:

$$
\begin{aligned}
s_{0} & =0.0001 \\
\bar{U}_{i} & =1.00 \\
\bar{L}_{i} & =0.05 \\
s_{i} & =0.8 .
\end{aligned}
$$

More detail of these parameters can be found in Svanberg (1987). These values were appropriate for our example problem as they made asymptotes close to iteration points and thus the program became more stable. This was necessary when the 


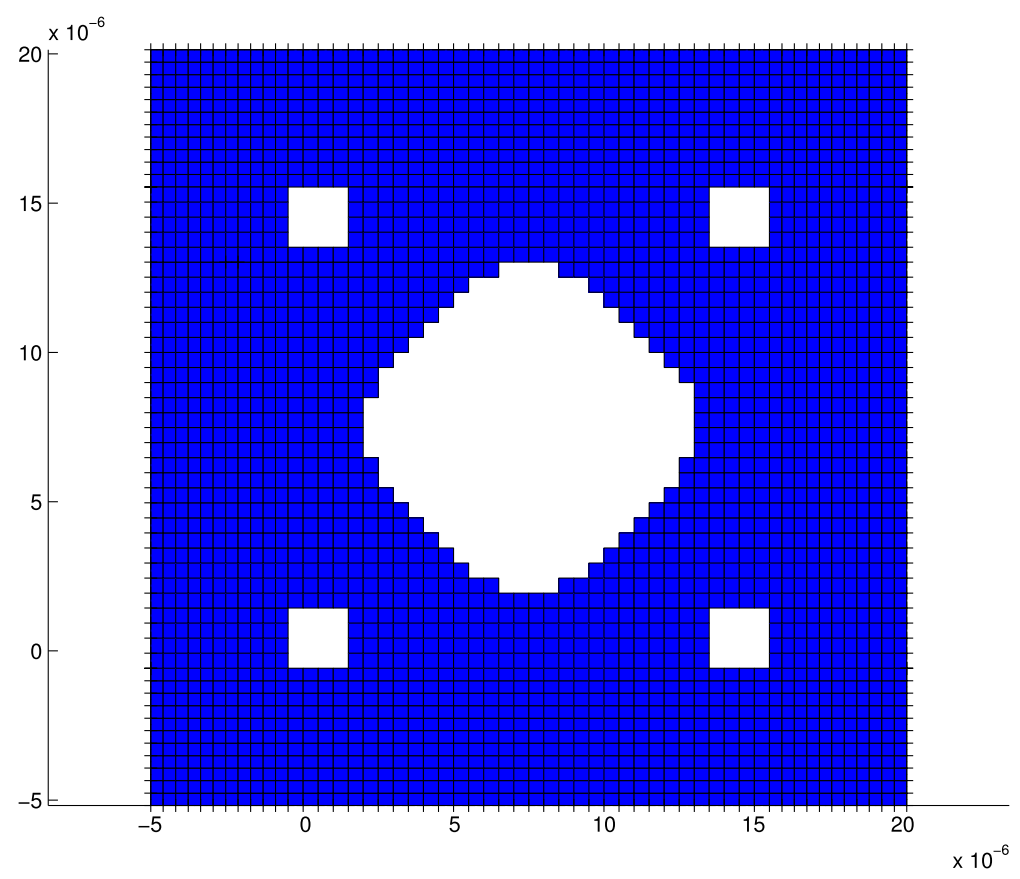

Fig. 9 Optimal topology for setting fundamental eigen-frequency to $1.4 \mathrm{GHz}$ by MMA. Dimensions are in meters

objective function value changed rapidly. For each value of $\epsilon, 100$ iterations of MMA were executed to solve the corresponding constrained problem. In the next main iteration $\epsilon$ was decreased by a factor of 0.8 (i.e., $\epsilon_{i+1}=0.8 \times \epsilon_{i}$ with $i$ denoting main iteration number). The process was repeated until both the objective function value and the constraint function value became small enough. As the starting point of optimization, the material density was uniform over the whole domain and roughly equal to 0.5 .

Figure 9 shows an optimal topology for setting the fundamental eigen-frequency. The target eigen-frequency is $1.5 \mathrm{GHz}$ and the structure obtained has a fundamental eigen-frequency of $1.4 \mathrm{GHz}$. Figure 10 shows an optimal topology for clustering the first two eigen-frequencies around $1.04 \mathrm{GHz}$ and we obtained $\omega_{1}=\omega_{2}=1.03 \mathrm{GHz}$. The corresponding eigen-modes are displayed in Figs. 11 and 12. Figure 13 shows the case where the first two eigen-frequencies are clustered around $1.04 \mathrm{GHz}$ and well separated from the third one. The first five eigen-frequencies for this topology are

$$
\begin{aligned}
& \omega_{1}=\omega_{2}=1.0230 \mathrm{GHz}, \quad \omega_{3}=1.2390 \mathrm{GHz}, \\
& \omega_{4}=1.6879 \mathrm{GHz}, \quad \omega_{5}=1.9348 \mathrm{GHz} .
\end{aligned}
$$




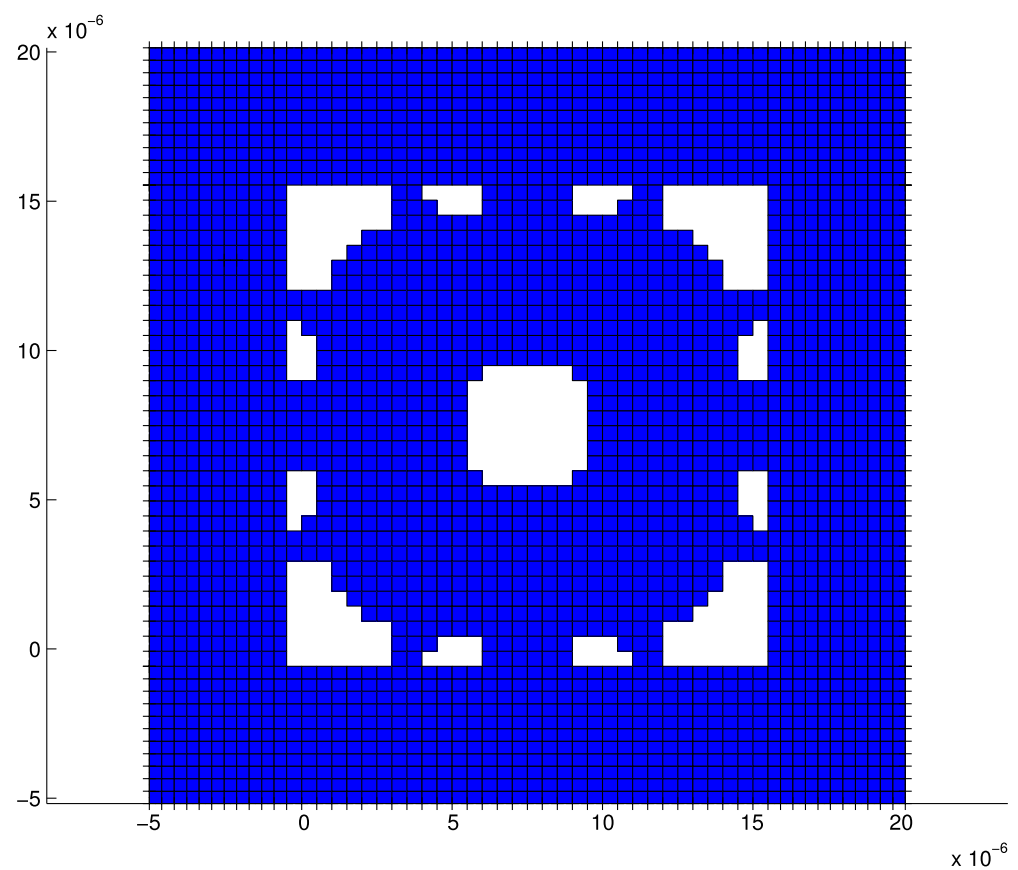

Fig. 10 Optimal topology for clustering the first 2 eigen-frequencies around $1.04 \mathrm{GHz}$ by MMA. Dimensions are in meters

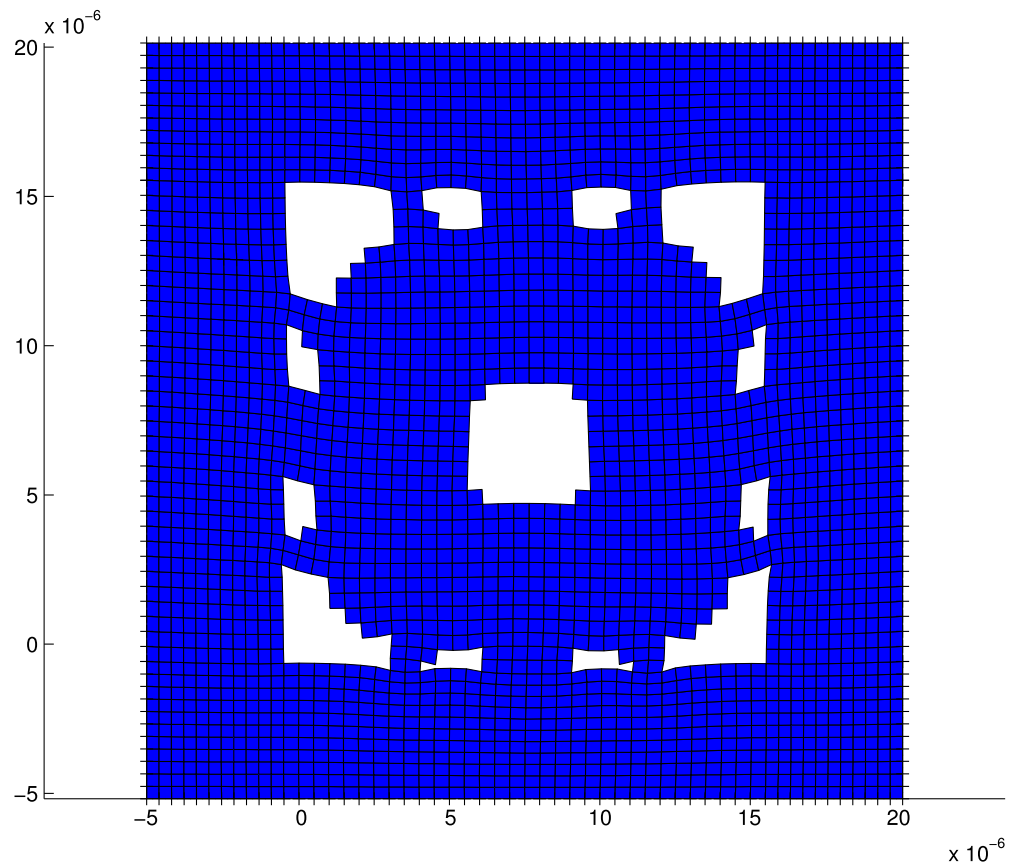

Fig. 11 First eigen-mode of structure in Fig. 10 


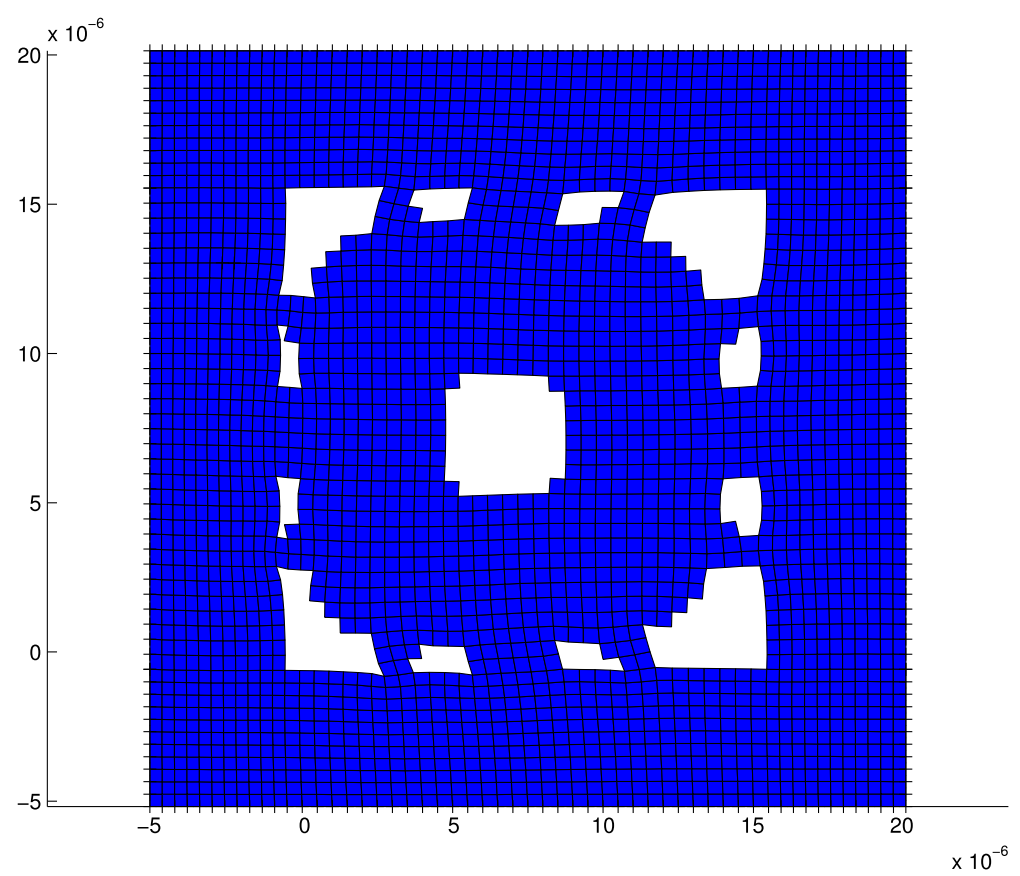

Fig. 12 Second eigen-mode of structure in Fig. 10

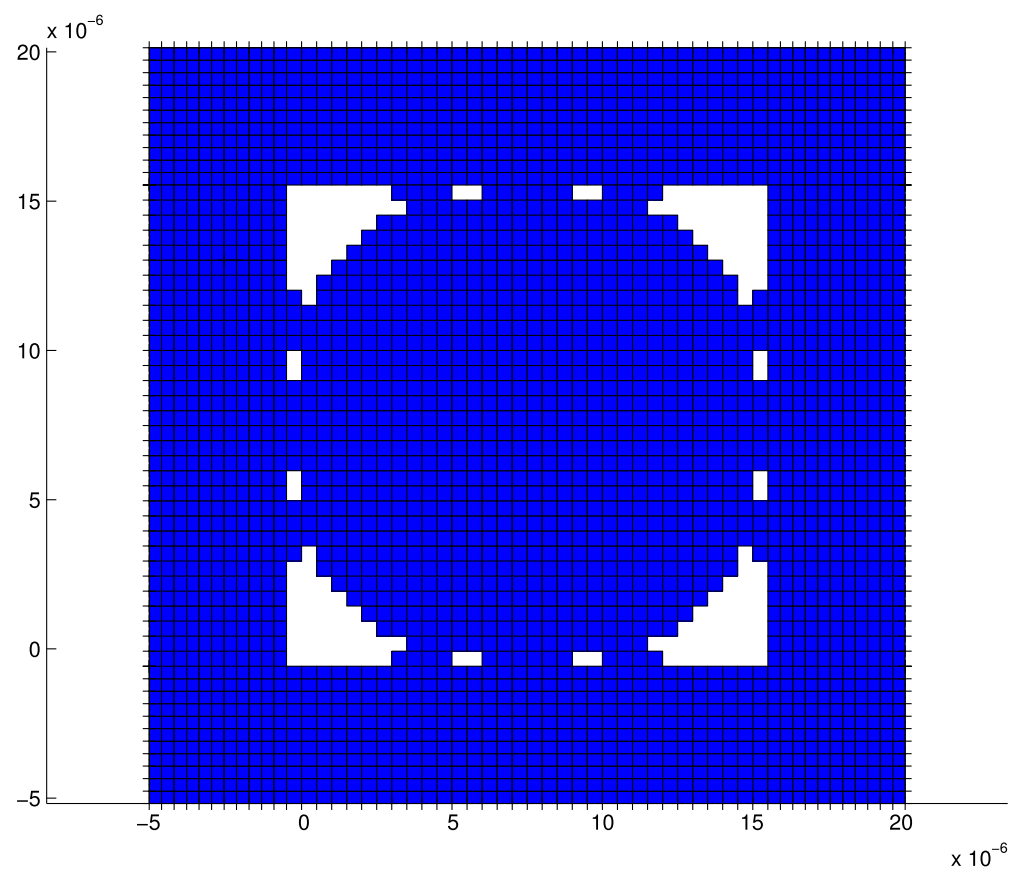

Fig. 13 Optimal topology for clustering the first 2 eigen-frequencies around $1.04 \mathrm{GHz}$ and separating from others. Dimensions are in meters 


\section{Summary and recommendations}

We have presented a few options for topological optimization for real eigenfrequencies of micromechanical resonators. Our focus on real eigen-frequencies was motivated by other work (Bindel and Govindjee 2005) showing that the clustering behavior of the real parts of the eigen-frequencies strongly governs resonator performance as measured by quality factors. The methods investigated are very easy to implement and are also applicable to other similar problems. As the first material distribution model, the peak function model was applied and the resulting optimization problem was solved using a Hybrid Genetic Algorithm. Local refinement was conducted using MATLAB's built-in function "fminunc" which implements the BFGS algorithm. The peak function model does not need side constraints on design variables, so we only need to solve an unconstrained optimization problem in local refinement and this improves efficiency. Hybrid GA is a global optimization method and can lead to better results in terms of objective function values but the resulting structures are usually irregular due to its stochastic characteristic. To avoid the irregularity of the resulting structures, one may consider imposing constraints on the perimeter of the structure (Harber et al. 1994, 1995, 1996; Beckers 1999; Duysinx 1997) or on the gradient of the density of material (Petersson and Sigmund 1998). The basic idea here is to achieve a well-posed mathematical formulation for topology optimization by further restricting the design space and removing rapid irregular oscillations in material densities.

As the second material distribution model, the SIMP model was also applied and the resulting problem was solved using MMA. MMA can usually find an optimal structure with some regularities. Thus for our problem class, we find the MMA algorithm to be superior, especially when the first-order derivatives of objective and constraint functions can be evaluated analytically. However, some techniques must be applied to avoid the nearest local minimum since MMA is essentially a local optimization algorithm. The technique used here is a heuristic homotopy method. The constraint is relaxed at the start, and then is gradually made strict. As a result, many (non-feasible) intermediate "densities" appear in the beginning of the optimization process, but this allows the algorithm to avoid local minima and achieve better results to some extent. As the feasible domain becomes exact, all the intermediate "densities" approach their limiting values of either 0 or 1 .

Open Access This article is distributed under the terms of the Creative Commons Attribution Noncommercial License which permits any noncommercial use, distribution, and reproduction in any medium, provided the original author(s) and source are credited.

\section{References}

Beckers M (1999) Topology optimization using a dual method with discrete variables. Struct Optim 17:1424

Bendsoe M (1989) Optimal shape design as a material distribution problem. Struct Optim 1:193-202

Bendsoe M (1995) Optimization of structural topology, shape, and material. Springer, Berlin

Bendsoe M, Kikuchi N (1988) Generating optimal topologies in structural design using a homogenization method. Comput Methods Appl Mech Eng 71:197-224 
Bindel D (2005) HiQLab: simulation of resonant MEMS. http://www.cims.nyu.edu/ dbindel/hiqlab/

Bindel D, Govindjee S (2005) Elastic PMLs for resonator anchor loss simulation. Int J Numer Methods Eng 64:789-818

Bruyneel M, Duysinx P, Fleury C (2002) A family of MMA approximations for structural optimization. Struct Multidiscip Optim 24:263-276

Chen S, Pan H (1986) Design sensitivity analysis of vibration models by finite element perturbation. In: Proceedings of the 4th international modal analysis conference, Los Angeles, California, USA, pp 38-43

Dailey R (1989) Eigenvector derivatives with repeated eigenvalues. AIAA J 27:486-491

Diaz AR, Kikuchi N (1992) Solution to shape and topolog eigenvalue optimization problems using homogenization method. Int J Numer Methods Eng 35:1487-1502

Duysinx P (1997) Layout optimization: a mathematical programming approach. DCAMM report 540, Dept of Solid Mechanics, Technical of Denmark

Fleury C (1989) Efficient approximation concepts using 2nd order information. Int J Numer Methods Eng 28:2041-2058

Fleury C, Braibant V (1986) Structural optimization-a new dual method using mixed variables. Int J Numer Methods Eng 23:409-428

Harber R, Jog C, Bendsoe M (1994) Variable-topology shape optimization with a control on perimeter. Adv Des Autom 69:261-272

Harber R, Jog C, Bendsoe M (1995) The perimeter method-a new approach to variable-topology shape optimization. In: Olhoff N, Rozvany G (eds) Proceeding of WCSMO-1, structural and multidisciplinary optimization. Elsevier Science, Oxford, pp 153-160

Harber R, Jog C, Bendsoe M (1996) A new approach to variable-topology design using a constraint on the perimeter. Struct Optim 11:1-12

Hashin Z, Shtrikman S (1963) A variational approach to the theory of the elastic behaviour of multiphase materials. J Mech Phys Solids 11:127-140

Hilding D (2000) A heuristic smoothing procedure for avoiding local optima in optimization of structures subject to unilateral constraints. Struct Multidiscip Optim 20:29-36

Houck C, Joines J, Kay M (1995) A genetic algorithm for function optimization: a Matlab implementation. Technical report NSCU-IE, North Carolina State University, Raleigh, NC

Juang J, Ghaemmaghami P, Lim K (1989) Eigenvalue and eigenvector derivatives of a nondefective matrix. J Guidance 12:480-486

Kenny S, Hou G (1994) Approximate analysis for repeated eigenvalue problems with applications to controls-structures integrated design. NASA technical paper 3439

Kosaka I, Swan CC (1999) A symmetry reduction method for continuum structural topology optimization. Comput Struct 70(1):47-61

Li S, Lin Y, Ren Z, Nguyen C (2007) An MSI micromechanical differential disk-array filter. In: Dig of tech papers, 14th int conf on solid-state sensors and actua tors, Transducers 07, pp 307-311

Ma Z, Hagiwara I (1991) Sensitivity analysis-methods for coupled acoustic-structural systems part I: modal sensitivities. AIAA J 29:1787-1795

Ma Z, Hagiwara I (1994) Development of new mode-superposition technique for truncating lower and/or higher-frequency modes (application of eigenmode sensitivity analysis for systems with repeated eigenvalues). JSME Int J Ser C 37(1):7-13

Ma Z, Kikuchi N, Cheng H, Hagiwara I (1995) Topological optimization technique for free-vibration problems. J Appl Mech 62:200-207

Ma Z, Kikuchi N, Hagiwara I (1993) Structural topology and shape optimization for a frequency-response problem. Comput Mech 13:157-174

Mills-Curran W (1988) Calculation of eigenvector derivatives for structures with repeated eigenvalues. AIAA J 26:867-871

Pedersen NL (2000) Maximization of eigenvalues using topology optimization. Struct Multidiscip Optim 20:2-11

Petersson J, Sigmund O (1998) Slope constrained topology optimization. Int J Numer Methods Eng 41:1417-1434

Poulsen T (2002) A simple scheme to prevent checkerboard patterns and one-node connected hinges in topology optimization. Struct Multidiscip Optim 24:396-399

Saxena A, Ananthasuresh GK (2000) On an optimal property of compliant topologies. Struct Multidiscip Optim 19:36-49

Sigmund O (1997) On the design of compliant mechanisms using topology optimization. Mech Struct Mach 25(4):493-524 
Sigmund O, Petersson J (1998) Numerical instabilities in topology optimization: a survey on procedures dealing with checkerboards, mesh-dependencies and local minima. Struct Optim 16:68-75

Suzuki K, Kikuchi N (1991) A homogenization method for shape and topology optimization. Comput Methods Appl Mech Eng 93:291-318

Svanberg K (1987) The method of moving asymptotes - a new method for structural optimization. Int J Numer Methods Eng 24:359-373

Svanberg K (1995) A globally convergent version of MMA without linesearch. In: Rozvany GIN, Olhoff N (eds) Proc. first world congress of structural and multidisciplinary optimization. Pergamon, Oxford, pp 9-16

Tcherniak D (2002) Topology optimization of resonating structures using SIMP method. Int J Numer Methods Eng 54:1605-1622

Yin L, Ananthasuresh G (2001) Topology optimization of compliant mechanisms with multiple materials using a peak function material interpolation scheme. Struct Multidiscip Optim 23:49-62

Yin L, Yang W (2000) Topology optimization for tunnel support in layered geological structures. Int J Numer Methods Eng 47:1983-1996 